\title{
Frühjahrstagung des Arbeitskreises Entwicklungspolitik und humanitäre Hilfe der DeGEval
}

\author{
\#ZahlenDatenFakten - Monitoring in der \\ Entwicklungszusammenarbeit und der humanitären \\ Hilfe zwischen Anspruch und Realität
}

\author{
Alexander Chenchenko, ${ }^{1}$ Susanne von Jan, ${ }^{2}$ Thorsten Bär, ${ }^{1}$ \\ Kirsten Vorwerk ${ }^{3}$
}

Monitoring ist ein wichtiger Aspekt bei der Durchführung von Projekten der Entwicklungszusammenarbeit und humanitären Hilfe. Monitoring wird häufig verstanden als „die regelmäßige Erhebung von Informationen mit dem Ziel, die Fortschritte des Projekts gegenüber der Planung (...) zu überprüfen“ (Kurz/Kubek 2015: 49). Im Monitoring können verschiedene Aspekte betrachtet werden, zum Beispiel die Nutzung des Inputs, die Durchführung der Leistungen und ihre Qualität, die Erreichung von direkten und gegebenenfalls indirekten sowie intendierten und nicht intendierten Wirkungen. Weiterhin kann sich das Monitoring auf ein bestimmtes Projekt oder ein Programm beziehen oder auch als institutionelles Monitoring einen Blick auf die jeweilige Organisation werfen. Ziel der diesjährigen Frühjahrstagung des Arbeitskreises Entwicklungspolitik und humanitäre Hilfe der Gesellschaft für Evaluation (DeGEval), welche im März 2020 in Bonn beim DAAD (Deutschen Akademischen Austauschdienst) ausgerichtet wurde, war es, zu diskutieren, welche Anforderungen und Standards an Monitoring gestellt werden können und inwiefern Monitoring den unterschiedlichen Anforderungen gerecht werden kann. Dazu trugen insgesamt 100 Teilnehmende im gemeinsamen Erfahrungsaustausch bei.

Als Gastgeberin begrüßte die Direktorin der Abteilung Strategie vom DAAD, Christiane Schmeken, die Teilnehmenden herzlich und leitete in einer kurzen Vorstellung über die Bedeutung des wirkungsorientierten Monitorings für die Arbeit des DAAD in die Thematik der Frühjahrstagung ein.

Masahiro Igarashi von der Food and Agriculture Organization of the United Nations (FAO) führte als Keynote-Referent in die Thematik des Monitorings ein. Er

World Vision Deutschland, Friedrichsdorf

2 smep-consult, Salzburg

3 Deutsches Evaluierungsinstitut der Entwicklungszusammenarbeit (DEval), Bonn 
beleuchtete in seinem Vortrag wie Definitionen, Abgrenzungen, Qualitätsanforderungen und Standards in Bezug auf Monitoring verstanden werden können. Der Komplexität von Monitoring kann durch das Identifizieren von Hauptfaktoren und wechselseitigen Beziehungen unter anderem durch das Aufstellen von ,System-Maps ${ }^{6}$ begegnet werden. Monitoring sollte dabei in ein organisationales Wissensmanagement eingebunden sein. Wenn es um die Art der Datenerhebung im Monitoring geht, so sollten neben quantitativen Methoden auch qualitative Daten erhoben werden, welche die spezifischen Fragen des Wie? und Warum? beantworten. Hierbei betonte Igarashi die Bedeutung von angemessenen und nützlichen Analyse- und Dokumentationsmethoden. Quantitative und qualitative Daten sollen den Projektmitarbeitenden schnell digital zur Verfügung stehen. Abschließend ging der Referent auf die zunehmende Bedeutung von Monitoring im Rahmen von Projekten im Nexus zwischen humanitärer Hilfe und Entwicklungszusammenarbeit ein. Hier sei die Nutzung von Monitoringdaten für ,Adaptive Programming' besonders wichtig, um einerseits Monitoringdaten schnell für Projektsteuerung nutzen zu können und andererseits mögliche Risikoszenarien im Blick zu haben.

An den Vortrag von Masahiro Igarashi anschließend wurden die Teilnehmenden der Frühjahrstagung in Kleingruppen (jeweils entweder Evaluierende/Beratende oder Vertreter(innen) von Organisationen, die Projekte umsetzen) eingeteilt, um Mind-Maps und Poster zu erarbeiten. Die Gruppen bearbeiteten drei Leitfragen und stellten die Ergebnisse in kurzen Vorträgen den jeweils anderen Gruppen gebündelt vor. Die zu beantwortenden Fragen lauteten wie folgt: (1) Welche Standards und Gütekriterien sind Ihres Erachtens für Monitoring besonders wichtig? (2) Welche Herausforderungen gibt es bei der Umsetzung des Monitorings, insb. hinsichtlich dieser Standards und Gütekriterien? (3) Welche Tipps und Empfehlungen haben Sie? Die Interaktion zwischen den unterschiedlichen Gruppen förderte nicht nur den Austausch untereinander, sondern regte auch zur Reflexion des Vortrags von Masahiro Igarashi an. Die Ergebnisse der Gruppenarbeiten sind in der Dokumentation der Veranstaltung auf der DeGEval Internetseite des Arbeitskreises zu finden. ${ }^{4}$

Im Anschluss an den Überblick zu den Gütekriterien und Standards von Monitoring wurde der Fokus auf methodische Ansätze des Projektmonitorings gelegt. So stellten Felipe Isidor-Serrano und Olaf Haasner (MainLevel) in ihrem Vortrag dar, inwiefern der Anspruch und die Realität von Projektmonitoring mit der Theorie des digitalen Denkens zu verbinden ist. Zu Beginn des Vortrags erörterten beide Referenten, dass Monitoring im Kontext der Entwicklungszusammenarbeit politisch durch die Paris Declaration und die SDGs verankert ist. Diese politische Verankerung habe die Qualität des Monitorings in der Entwicklungszusammenarbeit positiv beeinflusst. In der Praxis blieben jedoch viele Monitoringsysteme hinter den Erwartungen zurück. Die Referenten beziehen sich dabei auf zwei Herausforderungen: Einerseits müsse sich die Frage gestellt werden, ob Bezüge zu Indikatoren und Monitoringplänen für die politische Kommunikation ausreichend sind und andererseits, inwiefern erhobene Monitoringdaten zur Projektsteuerung genutzt werden können.

4 Ebenso können die einzelnen Präsentationen der Vorträge der Referierenden auf folgender Website eingesehen werden: https://www.degeval.org/de/arbeitskreise/entwicklungspolitik-und-huma nitaere-hilfe/aktuelles/ [29.06.2020]. 
In der weiteren Präsentation gingen die Referenten v.a. auf die Verknüpfung zwischen Monitoring und Projektsteuerung ein. Die Gründe für Herausforderungen in diesem Bereich sind vielfältig: Unterschiedliches Verständnis von Indikatoren, keine Daten(-quellen) zu Indikatoren, der Eindruck „M\&E sei für die Geber“ und daraus resultierend eine Trennung von Projektsteuerung und Monitoringsystemen. Laut den Referenten kann digitales Denken die in der Projektarbeit häufig aufkommenden fehlenden Verknüpfungen zwischen Indikatoren und Daten herstellen. Indem die Datenstruktur schon bei der Planung mitbedacht wird, wird eine effektive Operationalisierung von Indikatoren ermöglicht. Durch ein solches Vorgehen im Sinne des digitalen Denkens könne Monitoring und Projektsteuerung enger miteinander verbunden werden.

An diesen Vortrag anschließend führte Ulrich Schmidt von der Gesellschaft für Zwischenlagerung (BGZ) in die Grundlagen des Wissensmanagements ein. In seiner Präsentation erörterte er zunächst den systematischen Wissenstransfer, durch welchen eine Organisation einen Fokus auf Dokumentation und Zirkulation von Informationen und Erfahrungen legen kann. Dies bedeute jedoch auch, dass das Kreieren von Innovation oft nicht im Fokus des Wissensmanagements steht. Innovationen sind jedoch essenziell für bestimmte Organisationen, um aus Prozessen des Wissensmanagements selbst Lehren ziehen zu können. Ein weiterer Kritikpunkt am systematischen Wissenstransfer ist, dass Barrieren, welche die Anwendung von Wissen verhindern, nicht betrachtet werden. Um diese Barrieren der Anwendung von Wissen im Blick zu haben und den Fokus auf essenzielle Innovationen zu richten, stellte Ulrich Schmidt sieben Maximen für einen erfolgreichen Umgang mit Wissen vor. Anders als beim systematischen Ansatz, bei welchem Wissen im Sinne eines Erkenntnisstands eingeordnet wird, zeigte Ulrich Schmidt mit den sieben Maximen, dass Wissensmanagement im Sinne eines Lösungswegs verstanden werden kann. Als Lösungsweg gesehen, unterstützt Wissensmanagement eine wissensorientierte Unternehmensorganisation im Sinne einer lernenden Organisation.

Mit ihrem Vortrag über wirkungsorientiertes Monitoring auf institutioneller Ebene erörterten die Vortragenden Ruth Fuchs, Daniel Lechner und Mareike Fehling vom Deutschen Akademischen Austauschdienst (DAAD) Herausforderungen und Lösungsansätze des wirkungsorientierten Monitoringsystems des DAAD. Dabei erläuterten die Vortragenden Anforderungen des DAAD an ein institutionelles Monitoring, wie eine erhöhte Transparenz und das gemeinsame Lernen zwischen Geldgeber(inne)n, DAAD und Hochschulen. Durch eine wirkungsorientierte Programmplanung werden Zieldefinitionen und Hypothesen von Anfang an mitbedacht. So können auch Indikatoren auf Projektebene von den Hochschulen selbst definiert werden. Dadurch wird den Hochschulen ein größerer Gestaltungsspielraum gewährleistet. Durch das wirkungsorientierte Monitoring konnten Standards gesetzt werden, welche auch für das Evaluationssystem relevant sind. So sollen für Monitoring und Evaluierung die gleiche Systematik für Wirkungserfassung (ausgehend vom Wirkungsgefüge) genutzt sowie Synergieeffekte erzielt werden. An dieser Stelle wiesen die Referierenden auch auf die Wichtigkeit von verbindlichen Standards und Qualitätskriterien hin. Ebenso stellten die Referierenden Lernerfahrungen und Erfolgsfaktoren dar. Da es sich um einen komplexen Veränderungsprozess auf Ebe- 
ne der Gesamtorganisation handelt, ist es insbesondere wichtig, eine klare Steuerungsstruktur herzustellen und Rollen sowie Verantwortlichkeiten zu definieren. Ebenso ist es von großer Bedeutung, passgenaue IT-Lösungen miteinzubeziehen. Ein weiterer Erfolgsfaktor ist der kontinuierliche Dialog mit den Stakeholdern (z.B. Geldgeber(inne)n, Hochschulen und Partner(inne)n). Als Herausforderungen für das Projekt sehen die Referierenden einerseits die Bereitstellung von Ressourcen für Umsetzung und Weiterentwicklung des Systems und andererseits die Integration des Monitorings in Standardprozesse des gesamten DAAD.

Zum Abschluss des ersten Tages der Frühjahrstagung leitete Susanne von Jan, Sprecherin des Arbeitskreises, eine Fishbowl-Diskussion zum Thema ,Anspruch und Umsetzbarkeit von Monitoring'. In dieser Diskussion wurden die wichtigsten Aspekte des ersten Tages zusammengetragen und mit dem Plenum diskutiert. Es wurden insgesamt 3 Fragen gestellt, zu denen die Teilnehmenden eigene Erfahrungen und Beispiele präsentieren konnten. Die Fragen lauteten: Wie können qualitative Daten im Monitoring Verwendung finden? Wie kann die Rechtzeitigkeit qualitativ hochwertiger Daten gewährleistet werden? Wie gelingt es, Ownership und Empowerment der Zielgruppen durch Monitoring zu fördern?

In der Diskussion wurde unter anderem festgestellt, dass durch einen Fokus auf qualitative Daten ein stärkerer Bezug zur Projekt- und Feldebene hergestellt werden kann und Narrative beispielsweise für die politische Kommunikation besser genutzt werden können. Zudem wurde hervorgehoben, dass die Daten eingeordnet und kontextualisiert werden müssen. Um die Rechtzeitigkeit qualitativ hochwertiger Daten zu gewährleisten, wurde unter anderem empfohlen, moderne technische Lösungen im Monitoring zu nutzen, weil durch diese Zeit eingespart werden kann. Bei Evaluationen wurde der Zeitpunkt der Evaluationen als entscheidend bezeichnet, damit während eines noch laufenden Projekts ggf. Umsteuerungsmaßnahmen greifen können. Als letzter Punkt in der Diskussion wurde mit einem Beispiel erörtert, dass im besten Fall die Fähigkeit der Zielgruppen zur Selbststeuerung durch das Monitoring gefördert wird. Hierbei wurde ein stärkerer Fokus auf partizipative Methoden im Monitoring vorgeschlagen.

Der zweite Tag begann mit dem Vortrag zum Thema ,Nutzung von Monitoring und Lernen' von David Schmid (GIZ). Herr Schmid beleuchtete in seinem Vortrag verschiedene Dimensionen der Nutzung von Monitoring-Daten: In der tatsächlichen Anwendung rücke dabei oft die Rechenschaft gegenüber Geldgeber(inne)n an erste Stelle, danach positioniere sich die Projektsteuerung und erst an letzter Stelle sei der Bereich des Lernens aus Monitoring-Daten selbst zu verorten. In Bezug auf die Projektsteuerung gab David Schmid an, dass durch die komplexen und multidisziplinären Programme - welche sich teilweise auf fragile Kontexte beziehen - eine klassische Planung nur mit ,LogFrames' häufig nicht mehr ausreichend sei und sich deswegen vermehrt auf ,Adaptive Programming'-Ansätze fokussiert werden müsse. Inspiriert durch den Vortrag über Wissensmanagement von Ulrich Schmidt (GBZ) am Vortag zeigte David Schmid in Anlehnung an Kompetenzmodelle und Kompetenzstufenmodelle der GIZ auf, wie individuelles und institutionelles Lernen gefördert werden kann. Dabei werden verschiedene Entwicklungsstufen durchlaufen. Um das Lernen aus Monitoring-Daten zu fördern, plädierte David Schmid u.a. für einen 
stärkeren Fokus auf agile Ansätze, durch welche vor Allem die Nutzer(innen)perspektive besser im Blick gehalten werden könne (Human-Centered-Design). So könne beispielsweise durch die Äußerung von Bedürfnissen in Design-Workshops nicht nur die Nutzer(innen)perspektive besser verstanden werden, sondern auch das Monitoringsystem sich diesen Bedürfnissen im Voraus anpassen. Im letzten Teil des Vortrags zeigte David Schmid zudem verschiedene Ansätze der GIZ, welche sich dem Aspekt des Lernens aus Monitoring-Daten widmen: z.B. ,Blended-Learning'-Formate und integrierte digitale Anwendungen. Vor allem das Community-basierte Wissensmanagement nehme eine wichtige Rolle ein, da hier ein Fokus auf Peer-Learning und gegenseitigen Austausch gelegt wird.

Die Teilnehmenden konnten als nächsten Tagungspunkt zwischen drei Werkstattgesprächen wählen.

Das erste Werkstattgespräch befasste sich mit dem Zusammenhang zwischen Monitoring und humanitärer Hilfe unter der Moderation von Peter Hermes vom Auswärtigen Amt (AA). Thomas Rottland von CARE Deutschland stellte in seinem Vortrag Monitoring im Kontext der humanitären Hilfe am Beispiel von CAREs Arbeit in Nordwest-Syrien vor. Bereits zu Beginn des Vortrages machte Thomas Rottland deutlich, dass Monitoring in diesem Nothilfekontext sehr schwer durchzuführen ist. Große Herausforderungen für das Monitoring in diesem Umfeld sind unter anderem die Instabilität durch Kampfhandlungen samt der dadurch ausgelösten vielfältigen Fluchtbewegungen, die allgemeine Unsicherheit im Projektgebiet, das Remote-Management und die Arbeit mit vielen verschiedenen Partner(inne)n. Thomas Rottland beleuchtete im weiteren Verlauf des Vortrags die Vorgehensweise von CARE angesichts dieser Herausforderungen. Erstens werden stabile Implementierungsprozesse geschaffen, welche zusammen mit gezieltem Training dazu dienen sollen, Verantwortlichkeiten schnell zu delegieren und eine gute Koordination zu ermöglichen. Zweitens investiert CARE viele Ressourcen in die Qualität der Arbeit - dies spiegelt sich z.B. in der Größe der unterstützenden M\&E-Teams wider. Monitoring wird dabei oft weniger als Lerninstrument genutzt, sondern aufgrund der Gefahren und Umstände als Werkzeug, um die Fortschritte der Implementierung zu dokumentieren. Drittens geht CARE mit einem ,Multi-Layered-Monitoring'Ansatz vor: Monitoring wird hier durch verschiedene Stellen umgesetzt, z.B. durch Partnerorganisationen und CARE-Consultants, welche auf mehreren Ebenen und mit mehreren Ansätzen Monitoring in Nordwest-Syrien durchführen. Das Feedback der Begünstigten wird hierbei im Sinne eines Third-Party-Monitorings eingesetzt. Anschließend an den Vortrag von Thomas Rottland arbeiteten die Teilnehmenden des Werkstattgesprächs in einer Gruppenarbeit an drei Fragen das Thema des Vortrags weiter aus. Die Fragen lauteten dabei: Welche Anforderungen dürfen an Monitoring in der humanitären Hilfe gestellt werden, was ist zu viel verlangt? Wie wichtig sind qualitative Methoden in der humanitären Hilfe und wie können diese realistischerweise eingesetzt werden? Wie kann Partizipation im Monitoring der humanitären Hilfe angewendet werden, auch bei Remote-Ansätzen?

Das zweite Werkstattgespräch befasste sich mit dem Thema ,Erfahrungen zum Aufbau von Monitoring-Kapazitäten' unter der Moderation von Robert Heine (Energypedia-Consult). David Vossebrecher von der Rosa Luxemburg Stiftung 
(RLS) stellte in seinem Vortrag Grundprinzipien im Monitoring vor, beispielsweise, dass Monitoring auf Ebene der Projektumsetzung verankert ist und Ownership vor Ort fördern soll. Weiterhin zeigte er in seinen Überlegungen, dass nicht nur der Erfahrungshintergrund der Mitarbeitenden und Partnerorganisationen divers ist, sondern auch die heterogenen Standort-,Kulturen " sich in unterschiedlichen Arbeitsweisen spiegeln. Daraus entstehen verschiedene Herausforderungen für den Aufbau von Monitoring-Kapazitäten hinsichtlich des Wissens, Prozessen und Haltung gegenüber Monitoring. Als Ansätze und Lösungen für diese Herausforderungen empfahl David Vossebrecher einerseits die Erstellung von Leitfäden und Definitionen und andererseits die Kontextualisierung von Begriffen und Konzepten selbst. Eine weitere $\mathrm{Ma} ß n a h m e$ sei die Begleitung von Monitoring-Prozessen durch einen Pool externer Berater(innen). Zudem sei eine zukünftige Überlegung, eine Plattform für das Projektmanagement zu erstellen. Gegen Ende des Vortrags zeigte David Vossebrecher auf, dass aufgrund der vielen Facetten und Faktoren auch verschiedene kontextabhängige Lösungsansätze gesucht werden müssen und kein allgemeingültiges Rezept für alle gelten könne.

Britta Gentsch (GFA) und Julia Krömer (Europäische Komission/GFA) stellten in ihrem Vortrag ein noch bis 2021 laufendes Projekt mit dem Titel „Support to Design, Monitoring and Results Reporting for Projects and Programmes Financed by the European Union's External Assistance“ vor. In ihrem Vortrag gingen die Referentinnen darauf ein, dass es im Monitoring im EC DEVCO System drei Haupttypen gäbe: Monitoring verankert beim Projekt bzw. der Umsetzungsebene, Monitoring durch DEVCO Operational Manager, die für die operationale und finanzielle Supervision zuständig sind, sowie Monitoring durch externe Consultants (ROM). Die Vortragenden legten innerhalb ihrer Präsentation durch drei Beispiele dar, wie Monitoring Kapazitäten gestärkt werden können. Einerseits gibt es die Möglichkeit, Trainings für das interne Monitoring anzubieten, beispielsweise Face-to-Face-Trainings oder Webinare, in denen z.B. interne Monitoringprozesse gelehrt werden. Andererseits können Monitoring Kapazitäten gestärkt werden, indem Orientierungshilfen für das Erstellen von ,LogFrames' mit Zielformulierungen und Indikatoren gegeben werden. Eine weitere Möglichkeit besteht in der Bereitstellung neuerer ITSysteme für das Projektmanagement, durch welches eine direkte Kooperation, Interaktion und Austausch zwischen den jeweiligen Partnern gewährleistet werden kann. In ihrem Fazit wiesen auch Britta Gentsch und Julia Krömer auf die Bedeutung des Ownerships im Monitoring auf allen Ebenen der Organisation hin.

Das dritte Werkstattgespräch handelte von den Themen ,Wirkungsmodelle und Monitoring von Evaluierungsarbeit‘. Die Moderation für dieses Werkstattgespräch übernahm Claudia Gottmann vom BMZ. Der erste Vortrag von Dorothea GiesenThole (GIZ) stellte dar, dass Wirkungsmodelle, also visualisierte Theorien des Wandels, bei der GIZ die Grundlage des wirkungsorientierten Monitorings (WoM) sind. Um die erwarteten Wirkungen von Evaluierungen der GIZ im Gesamten zu erfassen und mittelfristig monitoren zu können, wurde ein entsprechendes Wirkungsmodell für Evaluierungen entwickelt. In ihrem Vortrag präsentierte Dorothea Giese-Thole diese Theorie des Wandels der GIZ mit den Ebenen Impact und Outcomes, geplanten Outputs und sieben Aktivitätentypen. Als Outputs wurden beispielweise metho- 
disch-hochwertige Evaluierungen genannt, auf der Outcome-Ebene z.B. evidenzgestützte Entscheidungen in den Vorhaben sowie Rechenschaftslegung und beim Impact eine verbesserte Wirksamkeit der Vorhaben. Um vom Wirkungsgefüge zum Monitoring zu kommen, sei zunächst die Klärung der Anforderungen an das WoMSystem zu klären, beispielweise durch die Einbindung von Beteiligten in Strategie- und Steuerungsentscheidungen. Zudem sei es wichtig, Wirkungshypothesen und Indikatoren für Ziele und Wirkungen zu formulieren, um Wirkungen messbar zu machen. Letztlich sei es notwendig, Monitoring-Instrumente zu erstellen, sowie eine Datenerhebung und Datenanalyse durchzuführen. Abschließend sollten diese Daten dann zur Steuerung, Rechenschaftslegung und für Wissensmanagement und Lernen genutzt werden.

Der zweite Vortrag in diesem Werkstattgespräch wurde von Line Winterhoff (DEval) zum Thema ,Monitoring der Evaluierungsarbeit des DEval' präsentiert. Hierbei erläuterte die Referentin zu Beginn das Wirkungsgefüge des DEval. Von der Output-Ebene sollen die Evaluierungen des DEval sowie weitere Outputs (z.B. Vorträge) zu verschiedenen Outcomes führen, z.B. sollen Erkenntnisse in die strategische Planung des BMZ eingehen oder vom DEval (weiter)entwickelte Designs und Methoden in anderen Evaluierungen genutzt werden. Der langfristige Impact dreht sich um die Verbesserung der Entwicklungszusammenarbeit. Um die Wirkungen der Evaluierungen monitoren zu können, muss im Rahmen der Konzeptionsphase einer Evaluierung die Wirkungslogik derselben erarbeitet werden. Hierbei werden unter anderem mögliche (un)intendierte Wirkungen sowie die Relevanz von Evaluierungsfragen für Zielgruppen betrachtet. In der Synthese-, Berichts- und Umsetzungsphase werden die erarbeiteten Outputs der Evaluierung, die intendierten DEval-Outcomes und die selbst wahrgenommene Nützlichkeit der Outputs dokumentiert. Als weiteren Schritt des Monitorings nannte die Referentin das Umsetzungsmonitoring der DEval-Empfehlungen 12 bis 24 Monate nach der Evaluierung. Im Anschluss an die beiden Vorträge bildeten sich vier Gruppen und bearbeiteten vier Felder der vorgestellten Wirkungsmodelle. Dabei wurde beispielweise diskutiert, wie ein genannter Outcome aus Sicht der Teilnehmenden realistisch gemessen werden könne.

Um die Frühjahrstagung zusammenzufassen und die verschiedenen Positionen und Diskussionen zu reflektieren, wurden die Teilnehmenden in der Abschluss-Session unter der Leitung von Susanne von Jan gebeten, in einer Online-Umfrage unter einer Vielzahl von Schlagworten, die im Verlauf der Tagung genannt wurden, zentrale Standards und Anforderungen, welche an Monitoring zu stellen sind, auszuwählen. Die wichtigsten ausgewählten Schlagworte waren dabei: ein angemessenes Aufwand-Nutzen-Verhältnis, Partizipation, Datenqualität, Nützlichkeit für das Lernen und, am häufigsten gewählt, Nützlichkeit für die Steuerung. In einer abschließenden Diskussion wurden diese Schlagworte von verschiedenen Positionen genauer beleuchtet und kontextualisiert: aus Perspektive der NRO, der Consultings und des DAAD. Hierbei wurden die folgenden Begriffe als besonders relevant hervorgehoben: Pragmatismus, Partizipation, Datenqualität, Nützlichkeit, Ethik und Empowerment.

Zusammenfassend lässt sich feststellen, dass sich innerhalb der Frühjahrtagung dem Thema Monitoring von vielen verschiedenen Perspektiven gewidmet 
wurde. So standen Fragen nach qualitativen Methoden und dem Nutzen von Monitoringsystemen im Fokus vieler Debatten. Besonders interessant waren auch die Diskussionen über Wissensmanagement und die Möglichkeiten des Lernens aus Monitoring-Daten. Auch die verschiedenen Aspekte des Monitorings in der Entwicklungszusammenarbeit und in der humanitären Hilfe wurden erörtert. Die diversen Gruppenarbeiten und die lernoffene Atmosphäre halfen dabei, die unterschiedlichen Gesichtspunkte des Monitorings zu verstehen und einen Blick über den eigenen Tellerrand hinaus zu ermöglichen. Eine zentrale Erkenntnis der Frühjahrstagung war, dass die Aspekte Pragmatismus, Partizipation, Datenqualität und Nützlichkeit in besonderem Maße zu beachten sind, obwohl es hier ggfs. zu Trade-Offs kommen kann. Zudem konnte ein Spannungsfeld ausgemacht werden zwischen dem Nutzen für Projektsteuerung und Lernen einerseits und der Kommunikation und Rechenschaft des Projektfortschrittes gegenüber Geber(inne)n andererseits. Darüber hinaus waren Empowerment und Rechenschaft gegenüber der lokalen Bevölkerung wichtige Themen.

\section{Literatur}

Kurz, Bettina/Kubek, Doreen (2015): Kursbuch Wirkung: Das Praxishandbuch für alle, die Gutes noch besser tun wollen; mit Schritt-für-Schritt Anleitungen \& Beispielen. Berlin: Phineo (3. Aufl.). 